\title{
Long-Term Endoscopic Follow-Up of Patients with Chronic Radiation Proctopathy after Brachytherapy for Prostate Cancer
}

\author{
Masahiro Ohtani, ${ }^{1}$ Hiroyuki Suto, ${ }^{1}$ Takuto Nosaka, ${ }^{1}$ Yasushi Saito, ${ }^{1}$ Yoshihiko Ozaki, \\ Ryoko Hayama, ${ }^{1}$ Tatsushi Naito, ${ }^{1}$ Kazuto Takahashi, ${ }^{1}$ Kazuya Ofuji, ${ }^{1}$ Hidetaka Matsuda, ${ }^{1}$ \\ Katsushi Hiramatsu, ${ }^{1}$ Tomoyuki Nemoto, ${ }^{1}$ Hiroki Shioura, ${ }^{2}$ Hirohiko Kimura, \\ Yoshitaka Aoki, ${ }^{3}$ Osamu Yokoyama, ${ }^{3}$ and Yasunari Nakamoto ${ }^{1}$ \\ ${ }^{1}$ Second Department of Internal Medicine, Faculty of Medical Sciences, University of Fukui, 23-3 Matsuokashimoaizuki, \\ Eiheiji-cho, Yoshida-gun, Fukui 910-1193, Japan \\ ${ }^{2}$ Department of Radiology, Faculty of Medical Sciences, University of Fukui, Fukui 910-1193, Japan \\ ${ }^{3}$ Department of Urology, Faculty of Medical Sciences, University of Fukui, Fukui 910-1193, Japan
}

Correspondence should be addressed to Yasunari Nakamoto; nakamoto-med2@med.u-fukui.ac.jp

Received 4 January 2016; Accepted 16 May 2016

Academic Editor: Patrick J. O’Dwyer

Copyright ( $) 2016$ Masahiro Ohtani et al. This is an open access article distributed under the Creative Commons Attribution License, which permits unrestricted use, distribution, and reproduction in any medium, provided the original work is properly cited.

Background. Chronic radiation proctopathy (CRP) is late toxicity and associated with morbidity. Aim. To investigate the predictors of prognosis in patients with CRP after brachytherapy (BT). Methods. One hundred four patients with prostate cancer were treated with BT or BT followed by external-beam radiotherapy (BT + EBRT). We retrospectively investigated the 5-year incidence of rectal bleeding and endoscopic findings of CRP using the Vienna Rectoscopy Score (VRS). Twenty patients with VRS $\geq 1$ were divided into the improved VRS group without treatment, unchanged VRS group, and treated group. The parameters associated with alteration of VRS were analyzed. Results. The incidence of rectal bleeding was $24 \%$. The risk of rectal bleeding was higher in patients treated with BT + EBRT compared to those treated with BT $(p<0.0001)$. The incidence of superficial microulceration was higher in the improved VRS group than in the unchanged VRS group $(p<0.05)$. The incidence of multiple confluent telangiectasia or superficial ulcers $>1 \mathrm{~cm}^{2}$ was higher in the treated group than in both the improved and unchanged VRS groups $(p<0.05)$. Conclusions. Patients treated with BT + EBRT have a high risk of CRP. Endoscopic findings were useful for prognostic prediction of CRP.

\section{Introduction}

Prostate cancer is the most commonly diagnosed cancer and the second-leading cause of cancer-related mortality in men over the age of 40 years in the United States [1], with the incidence of prostate cancer in Japan increasing in recent years [2]. For localized prostate cancer, radiation therapy is an effective treatment modality. Since the approval of ${ }^{125}$ I seed source in $2003,{ }^{125}$ I brachytherapy (BT) has become one of the standard treatment modalities for low- to intermediaterisk prostate cancer in Japan. In addition, a combination of $\mathrm{BT}$ and external-beam radiation therapy (EBRT) is used for intermediate- to high-risk prostate cancer.
Chronic radiation proctopathy (CRP) is a late gastrointestinal (GI) toxicity and one of the common adverse effects after radiation therapy. Histopathological features of CRP are ischemic endarteritis and fibrosis in the submucosa [3]. The symptoms of CRP include hematochezia, tenesmus, diarrhea, fecal incontinence, and defecatory urgency. The most common complaint is rectal bleeding. CRP is temporary and self-limiting in approximately $95 \%$ of all patients; however, patients with severe recurrent hemorrhage may require hospitalization and blood transfusions [3]. Furthermore, various treatment modalities have been reported for the management of CRP, including endoscopic argon plasma coagulation (APC) [4], dilute formalin topical treatment [5], 
hyperbaric oxygen (HBO) therapy [6], and sucralfate enema [7]. For the evaluation of CRP, endoscopic examination is the most important modality. On endoscopy, the rectal mucosa may show varying appearances, from telangiectasia (TE) to ulceration (UL). Although the relationship between the symptoms and endoscopic findings of CRP has been investigated, literature about long-term follow-up with endoscopic examination is limited [8]. A study has reported the endoscopic findings at 12 and 65 months after EBRT, showing improvement in TE [8]. However, the long-term endoscopic evaluation of CRP after BT has not been reported. Furthermore, the indicators of medical treatment including APC or HBO therapy for rectal bleeding caused by CRP are unclear. Therefore, the purpose of this study was to evaluate the risk and prognostic factors of rectal bleeding after BT for prostate cancer.

\section{Patients and Methods}

2.1. Patients. A retrospective medical chart review was performed on consecutive patients with prostate cancer underwent brachytherapy between May 2006 and September 2009 at University of Fukui Hospital. This study was approved by institutional review board of University of Fukui, with the IRB number of 20150119. The inclusion criteria consist of histological diagnosis of prostate cancer and treatment with BT alone or BT followed by EBRT boost. The exclusion criteria were subjects with incompletion of follow-up after BT and coexistence of prostate and rectal cancer. Patients were implanted with ${ }^{125}$ I seeds (OncoSeed; Nihon Medi-physics Co., Japan) by using modified peripheral loading techniques and a Mick applicator (Mick Radionuclear Instruments, Bronx, NY, USA). The prescribed dose was 144 Gy in patients undergoing BT only and $105 \mathrm{~Gy}$ in those undergoing BT + EBRT [9]. For combined therapy, BT was performed first, followed by EBRT 4-8 weeks after BT. EBRT was delivered using 10-MV X-rays in two or four fields, with a daily fraction dose of $1.8 \mathrm{~Gy}$, for 5 days per week, up to total dose of 45$55 \mathrm{~Gy}$.

2.2. Evaluation of Late Rectal Toxicity. After radiation therapy, patients were routinely followed up every 3 months to record GI toxicity due to radiotherapy. Rectal bleeding was graded according to the National Cancer Institute Common Terminology Criteria for Adverse Event (CTCAE) version 4.0 [10]. Rectal bleeding was defined as four instances of hematochezia in 4 weeks [11]. No rectal bleeding was defined as CTCAE grade 0 for evaluating the relationship between toxicity grade and endoscopic scoring. We evaluated the incidence of rectal bleeding after BT for 5 years and the factors associated with rectal bleeding, including age, extent of the primary tumor in TMN classification, use of antithrombotic therapy, presence or absence of diabetes mellitus, volume of the prostate gland, presence or absence of EBRT boost, number of seeds, and the use of neoadjuvant androgen depletion therapy (ADT). Tumor staging was performed according to the International Union Against Cancer TMN Classification of Malignant tumors 6th edition [12].
2.3. Evaluation of Endoscopic Findings of CRP. Several endoscopists performed colonoscopy or rectosigmoidoscopy by using the PCF-Q260AI (Olympus, Japan) for symptoms of CRP, as part of a health check-up or as an examination for fecal occult blood. The endoscopic findings of rectal mucosa were graded using the Vienna Rectoscopy Score (VRS), as reported by Wachter et al. [13]. In brief, endoscopic findings including TE, congested mucosa, UL, stricture, and necrosis were graded according to the following graduation system:

TE: grade 0 , none; grade 1 , single TE; grade 2 , multiple nonconfluent TE; and grade 3 , multiple confluent TE.

Congested mucosa: grade 0 , none; grade 1 , focal reddening of the mucosa combined with an edematous mucosa; grade 2, diffuse but nonconfluent reddening of the mucosa combined with an edematous mucosa; and grade 3, diffuse confluent reddening of the mucosa combined with an edematous mucosa.

UL: grade 0 , none; grade 1 , superficial microulceration $<1 \mathrm{~cm}^{2}$; grade 2 , superficial UL $>1 \mathrm{~cm}^{2}$; grade 3 , deep UL; and grade 4, fistula and perforation.

Stricture: grade 0 , none; grade 1 , more than two-thirds of the regular diameter; grade 2, one-third to twothirds of the regular diameter; grade 3 , less than onethird of the regular diameter; and grade 4, complete obstruction.

Necrosis: grade 0 , none, and grade 1 , necrosis.

Each of the five endoscopic parameters were summarized as VRS, from 0 to 5.

The relationship between the VRS and CTCAE grade was analyzed. Furthermore, the 5-year follow-up period was divided into the early period (up to the first 150 weeks after BT) and the late period of posttreatment (more than 150 weeks after BT and thereafter). Patients who had undergone endoscopy in both early and late period were classified into three groups based on their findings, the improved VRS group, the unchanged VRS group, or the treated group (treated with APC or HBO therapy), to evaluate changes of endoscopic findings and to determine their clinical characteristics.

2.4. Statistical Analysis. The distribution of clinical parameters for rectal bleeding and endoscopic parameters associated with rectal mucosal alteration was analyzed using the Student $t$-test and Fisher's exact test. The cumulative incidence of rectal bleeding was evaluated with the Kaplan-Meier method, and significant differences between treatment modalities were calculated by the log-rank test. The relationship between VRS and the CTCAE grade, obtained at the time of endoscopic examination, was analyzed by using Spearman's correlation test. All statistical analyses were performed using GraphPad Prism 6 (GraphPad Software, Inc., La Jolla, CA). Statistical significance was defined as $p<0.05$.

\section{Results}

3.1. Patient Characteristics. A total of 104 patients with localized prostate cancer underwent transperineal ${ }^{125}$ I prostate 
TABle 1: Patient characteristics.

\begin{tabular}{lc}
\hline Characteristic & \\
\hline Number of patients & 104 \\
Age, mean \pm SD & $68.1 \pm 6.6$ \\
Clinical stage, $n(\%)$ & \\
T1c & $81(77.8)$ \\
T2a & $16(15.4)$ \\
T2b & $4(3.8)$ \\
T2c & $3(2.9)$ \\
Use of antithrombotic drugs (\%) & $21(20.2)$ \\
Presence of DM (\%) & $12(11.5)$ \\
Volume of prostate, (cc) mean \pm SD & $25.6 \pm 8.3$ \\
Treatment & \\
EBRT boost (\%) & $53(51.0)$ \\
Median dose of EBRT (range) & $45(43-55.6)$ \\
Median number of seeds (range) & $70(40-100)$ \\
ADT (\%) & $39(37.5)$ \\
\hline
\end{tabular}

DM, diabetes mellitus; EBRT, external-beam radiotherapy; ADT, androgen deprivation therapy.

BT between May 2006 and September 2009 at our institute. The characteristics of these patients are shown in Table 1. The mean age was 68.1 years (range: $54-82$ years). According to TMN classification, 81 and 23 patients were classified as having $\mathrm{T} 1$ and $\mathrm{T} 2$ disease, respectively. Twenty-one patients $(20.2 \%)$ received antithrombotic drugs for coronary artery diseases or cerebral infarction. A total of 12 patients (11.5\%) were treated for diabetes mellitus. The mean prostate volume before RT estimated on ultrasonography was $25.6 \mathrm{~mL}$. Combined therapy with EBRT was performed in 53 (51.0\%) patients. The median dose of EBRT was $45 \mathrm{~Gy}$ (range: 43$55.6 \mathrm{~Gy}$ ) and median number of ${ }^{125} \mathrm{I}$ seeds was 70 (range: 40-100). Thirty-nine patients were treated with neoadjuvant ADT consisting of luteinizing hormone-releasing hormone agonists and antiandrogen.

3.2. Risk Factors of Rectal Bleeding after Radiotherapy for Patients with Prostate Cancer. Rectal bleeding was observed in 25 patients $(24 \%)$, with grade $1(12.5 \%)$ being the most common, followed by grade $2(4.8 \%)$ and grade $3(6.7 \%)$ (Table 2). Endoscopy was performed in $56(53.8 \%)$ patients totally and in 24 of 25 patients who experienced rectal bleeding symptoms of CTCAE grade $\geq 1$. Iron supplementation was prescribed for 9 patients with anemia due to rectal bleeding. Endoscopic APC and HBO therapy were administered to 5 and 2 patients, respectively (Table 2). All patients who received endoscopic APC or HBO therapy showed improvement in their rectal bleeding symptoms. The occurrence of rectal bleeding was $41.5 \%$ in the BT + EBRT group while it was $5.9 \%$ in the BT group, indicating a significant association of the presence or absence of EBRT with rectal bleeding of CTCAE grade $\geq 1(p<0.0001)$ (Table 3). No significant differences were observed in other factors. In a log-rank test based on the presence or absence of EBRT, a significantly higher occurrence of rectal bleeding was found in the BT + EBRT group compared to the BT group
TABLE 2: Incidence of rectal bleeding, endoscopic examination, and treatment.

\begin{tabular}{lc}
\hline & $n(\%)$ \\
\hline CTCAE rectal bleeding scale & $13(12.5 \%)$ \\
Grade 1 & $5(4.8 \%)$ \\
Grade 2 & $7(6.7 \%)$ \\
Grade 3 & $25(24.0 \%)$ \\
Total & \\
Endoscopic examination & $56(53.8 \%)$ \\
Number of patients who underwent endoscopy & 101 \\
Total number of endoscopy & 24 \\
$\quad$ Number of patients who underwent endoscopy & \\
in group of grade $\geq 1$ rectal bleeding & \\
Treatment & 9 \\
$\quad$ Iron supplementation & 5 \\
Endoscopic argon plasma coagulation & 2 \\
$\quad$ Hyperbaric oxygen therapy & \\
\hline
\end{tabular}

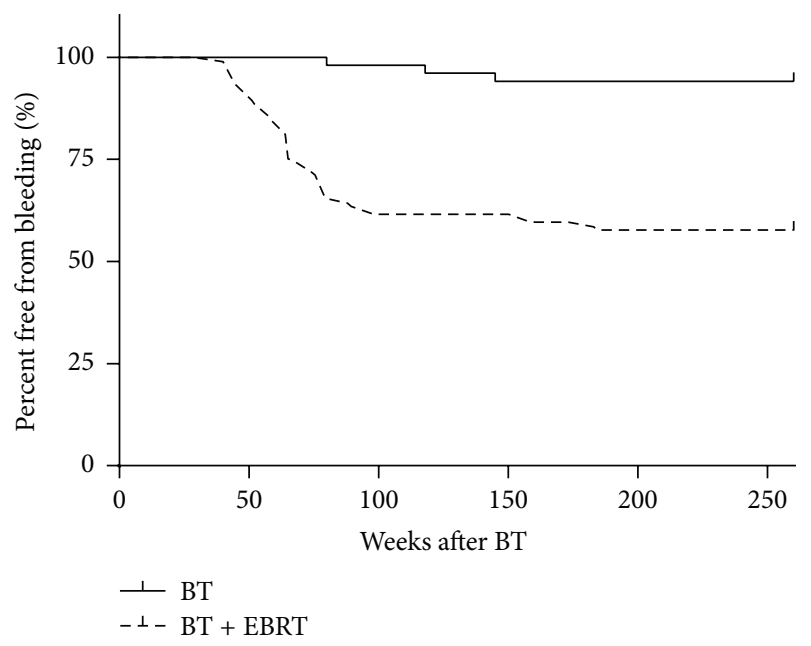

FIgURE 1: Kaplan-Meier curve for grade $\geq 1$ rectal bleeding over 5 years $(p<0.0001)$. BT: brachytherapy; EBRT: external-beam radiotherapy.

$(p<0.0001)$ (Figure 1), and the Kaplan-Meier curve showed that the first rectal bleeding occurred before the 150th week after BT in the BT group. Conversely, in the BT + EBRT group, rectal bleeding occurred more frequently in the earlier period after BT (50th to 100th week) compared to the BT group. These results suggest that EBRT boost after BT increased the risk of rectal bleeding.

3.3. Relationship between Endoscopic Findings and Rectal Bleeding Symptoms and Changes in Endoscopic Findings over Time. A total of 101 endoscopies were performed in the investigated patients during 5 years (Table 2). The VRS and CTCAE grade in patients who underwent endoscopies were significantly correlated ( $p<0.01$ ); CTCAE grade $\geq 1$ rectal bleeding was observed in $20.7 \%, 43.7 \%$, and $84.6 \%$ when 
TABLE 3: Clinical parameters associated with rectal bleeding.

\begin{tabular}{|c|c|c|}
\hline Parameter & Grade $\geq 1$ rectal bleeding (\%) & $p$ value \\
\hline \multicolumn{3}{|l|}{ Age, years } \\
\hline$\geq 70$ & $14 / 43(32.5)$ & \multirow{2}{*}{0.11} \\
\hline$<70$ & $11 / 61(18.0)$ & \\
\hline \multicolumn{3}{|l|}{ Stage } \\
\hline $\mathrm{T} 1$ & $19 / 81(23.4)$ & \multirow{2}{*}{0.79} \\
\hline $\mathrm{T} 2$ & $6 / 23(26.1)$ & \\
\hline \multicolumn{3}{|c|}{ Use of antithrombotic drugs } \\
\hline Yes & $7 / 21(33.3)$ & \multirow{2}{*}{0.27} \\
\hline No & $18 / 83(21.6)$ & \\
\hline \multicolumn{3}{|c|}{ Presence of DM } \\
\hline Yes & $2 / 12(16.6)$ & \multirow{2}{*}{0.73} \\
\hline No & $23 / 92(25.0)$ & \\
\hline \multicolumn{3}{|c|}{ Prostate volume, $\mathrm{cc}$} \\
\hline$\geq 25$ & $11 / 49(22.4)$ & \multirow{2}{*}{0.82} \\
\hline$<25$ & $14 / 55(25.4)$ & \\
\hline \multicolumn{3}{|c|}{ EBRT boost } \\
\hline Yes & $22 / 53(41.5)$ & \multirow{2}{*}{$<0.000$} \\
\hline No & $3 / 51(5.9)$ & \\
\hline \multicolumn{3}{|c|}{ Number of seeds } \\
\hline$\geq 70$ & $10 / 52(19.2)$ & \multirow{2}{*}{0.35} \\
\hline$<70$ & $15 / 52(28.8)$ & \\
\hline \multicolumn{3}{|c|}{ Use of ADT } \\
\hline Yes & $8 / 39(21.0)$ & \multirow{2}{*}{0.64} \\
\hline No & $17 / 65(10.8)$ & \\
\hline
\end{tabular}

DM, diabetes mellitus; EBRT, external-beam radiotherapy; ADT, androgen deprivation therapy.

VRS grade was 1,2 , and 3, respectively (Figure 2). A total of 52 endoscopies were performed in patients with CTCAE grade $\geq 1$. VRS 0 (normal) was found in $55.6 \%$ of the patients who underwent endoscopy within the first year after BT (0 to 1 year) (Figure 3). VRS 1 or higher was found in all patients who underwent endoscopy between 1 and 2 years after BT. Even though 7 patients who received APC or HBO therapy were included, the percentage of patients with VRS 1 increased with time (5 years) while the percentage of patients with VRS $\geq 3$ decreased (Figure 3 ). In patients with CTCAE grade 0,49 endoscopies were performed. The evaluation of VRS over time in patients without rectal bleeding revealed that VRS 0 was found in $88.9 \%$ of patients who underwent endoscopy between 0 years and 1 year after BT (Figure 4). In contrast, VRS $\geq 1$ was found in $80 \%$ of patients who underwent endoscopy between 0 years and 1 year after BT (Figure 4). With time, the percentage of patients with VRS 0 increased while the percentage of patients with VRS $\geq 2$ decreased in patients with CTCAE grade 0 . These results indicated that endoscopic findings were correlated with rectal bleeding symptoms, and abnormal findings that were frequently observed 1 year or longer after BT improved over time.

3.4. Prognostic Factors of CRP Based on Endoscopic Findings after Treatment. The changes in endoscopic findings in

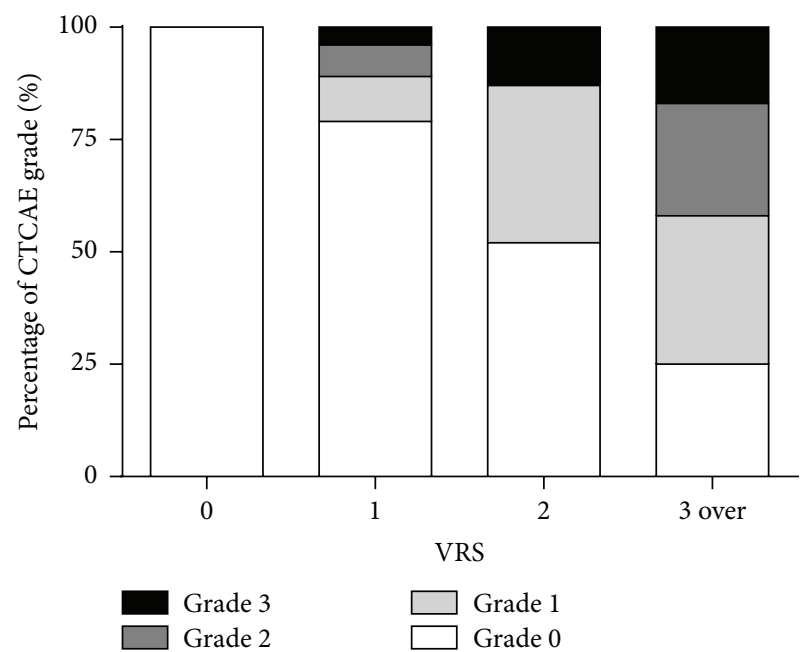

FIGURE 2: Correlation between VRS and CTCAE grade of rectal bleeding. The statistical significance was analyzed by Spearman's correlation test $(p<0.01)$. VRS: Vienna Rectoscopy Score.

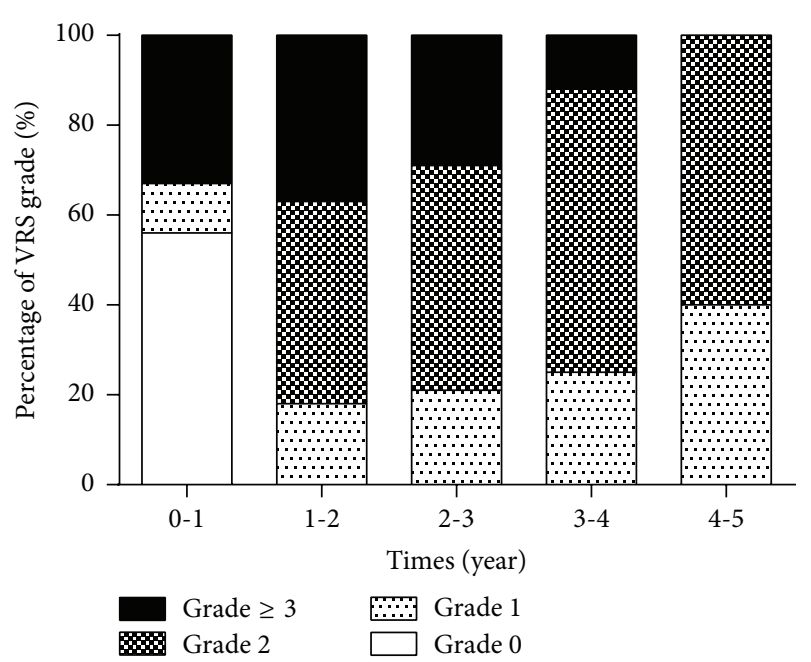

FIGURE 3: Change in VRS over time, in patients with CTCAE grade $\geq$ 1. VRS: Vienna Rectoscopy Score.

the early and late periods were evaluated in 26 patients. Six patients who underwent endoscopy in the early period after BT had VRS 0 , and these patients did not show any change in the endoscopic findings and bleeding symptoms in the late period.

Of 20 patients with VRS $\geq 1$ in the early period, 4,5 , and 11 patients were from the treated group with APC or HBO therapy, the improved VRS group without treatment, and the unchanged VRS group, respectively (Table 4). All patients with VRS 1 in the early period also did not show any change of VRS. However, improvement of VRS was observed in 2 of 10 patients with VRS 2 and 3 of 6 patients with VRS $\geq 3$. Rectal bleeding symptoms improved over the 5-year course in all patients of both the improved VRS and unchanged VRS groups. The percentage of patients with UL grade 1 was significantly higher in the improved VRS group (60\%) than 
TABLE 4: Endoscopic and clinical factors for improvement of VRS in patients with VRS $\geq 1$ who underwent follow-up endoscopic examination.

\begin{tabular}{|c|c|c|c|c|}
\hline & Treated group (HBO or APC) & Improved group & Unchanged group & $p$ value \\
\hline$N$ & 4 & 5 & 11 & \\
\hline \multicolumn{5}{|l|}{ VRS } \\
\hline 1 & 0 & 0 & 4 & \\
\hline 2 & 1 & 2 & 7 & \\
\hline$\geq 3$ & 3 & 3 & 0 & \\
\hline \multicolumn{5}{|l|}{ TE score } \\
\hline 0 & 0 & 0 & 0 & \\
\hline 1 & 0 & 0 & 4 & \\
\hline 2 & 1 & 5 & 7 & \\
\hline 3 & 3 & 0 & 0 & \\
\hline \multicolumn{5}{|l|}{ UL score } \\
\hline 0 & 0 & 2 & 11 & \\
\hline 1 & 1 & 3 & 0 & \\
\hline 2 & 0 & 0 & 0 & \\
\hline 3 & 1 & 0 & 0 & \\
\hline UL $1(+)$ & $1 / 4$ & $3 / 5^{\mathrm{a}}$ & $0 / 7^{\mathrm{a}}$ & $<0.05^{\mathrm{a}}$ \\
\hline TE 3 or $\mathrm{UL} \geq 2$ & $3 / 4^{\mathrm{b}}$ & $0 / 5^{\mathrm{b}}$ & $0 / 5^{\mathrm{b}}$ & $<0.05^{\mathrm{b}}$ \\
\hline Age, years & $71.8 \pm 8.3$ & $67.2 \pm 3.1$ & $69.7 \pm 4.2$ & NS \\
\hline Stage T1/T2 & $2 / 2$ & $3 / 2$ & $6 / 1$ & NS \\
\hline Use of ADT & 2 & 2 & 5 & NS \\
\hline Use of antithrombotic drugs & 2 & 1 & 1 & NS \\
\hline$p$ volume, $\mathrm{mL}$ & $22.5 \pm 5.5$ & $25.1 \pm 7.0$ & $24.6 \pm 9.4$ & NS \\
\hline Use of EBRT boost & 4 & 5 & 7 & NS \\
\hline Number of seeds & $71.3 \pm 6.3$ & $61.0 \pm 13.8$ & $62.9 \pm 13.3$ & NS \\
\hline
\end{tabular}

VRS, Vienna Rectoscopy Score; HBO, hyperbaric oxygen therapy; APC, argon plasma coagulation; TE, telangiectasia; UL, ulceration; ADT, androgen deprivation therapy; EBRT, external-beam radiotherapy; NS, not significant.

${ }^{\mathrm{a}}$ The improved group versus the unchanged group, $p<0.05$.

${ }^{\mathrm{b}}$ The treated group versus the improved group; the treated group versus the unchanged group, $p<0.05$.

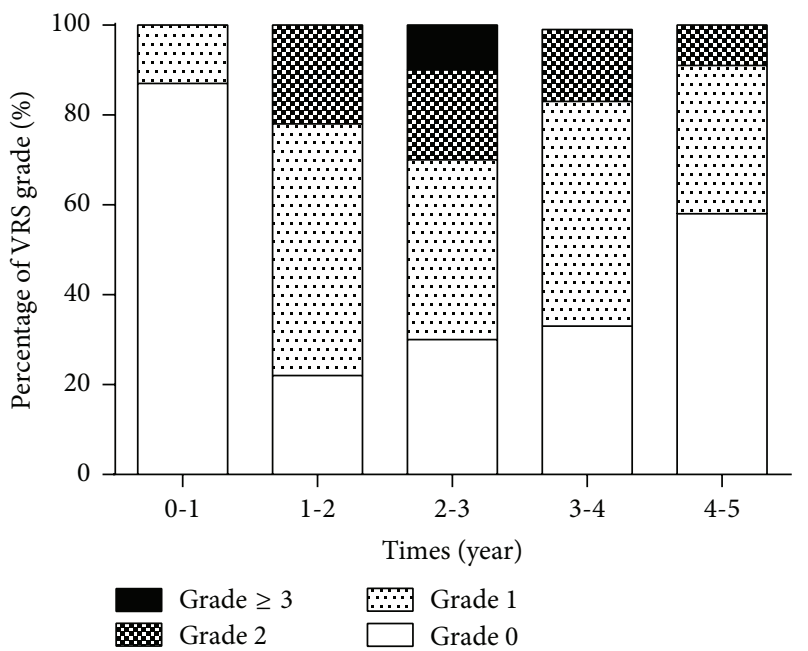

FIGURE 4: Change in VRS over time, in patients with CTCAE grade 0. VRS: Vienna Rectoscopy Score.

in the unchanged VRS group $(0 \%)(p<0.05)$ (Table 4$)$. When patients had TE grade 3 and UL grade $\geq 2$ along with higher endoscopic severity, $75 \%$ of patients in the treated group met the criteria while none satisfied the criteria in the improved VRS group and the unchanged VRS group $(p<$ 0.05) (Table 4). No differences were observed among the three groups considering the age; extent of the primary tumor $(\mathrm{T})$; use of neoadjuvant ADT, antithrombotic therapy; volume of the prostate gland; and the presence or absence of EBRT. These results indicated that natural recovery of superficial ulceration (UL grade 1) could be expected in patients with $\mathrm{VRS} \geq 2$.

\section{Discussion}

In this study, we examined risk factors of rectal bleeding and the prognosis of CRP after BT for prostate cancer. The risk of rectal bleeding significantly increased in the patients treated with BT + EBRT compared to those treated with BT alone. Most episodes of rectal bleeding were observed 1 year or more after BT, and endoscopic findings were correlated with the rectal bleeding score. Overtime evaluation of endoscopic findings after radiotherapy for 5 years revealed that spontaneous recovery was promising if UL grade was 1 or less in the first 150 weeks after BT.

Although the efficacy of radiotherapy for prostate cancer has been recognized, various late toxicities, including rectal 
bleeding, hematuria, and erectile dysfunction, have been reported, because of the rectum or the urethra adjacent to the prostate gland [14]. The occurrence of rectal bleeding after BT and BT + EBRT is 9.1-27.0\% and $18.3-47 \%$, respectively [15-18]. Although BT + EBRT was reported to increase rectal bleeding risk compared to $\mathrm{BT}[14,15]$, no difference in the incidence of toxicity between BT and BT + EBRT has been indicated [11, 18]. Therefore, the rectal bleeding risk and toxicity of these modalities are still controversial. Rectal bleeding risk increased in patients with coronary artery disease while ADT decreased the risk [11]. However, in the present study, no difference was observed in the use of antithrombotic therapy. The use of antithrombotic therapy also results in a rectal bleeding risk after BT or EBRT monotherapy $[16,19]$; hence, the limited number of patients in the present study was a possible reason for this difference in results compared to previous studies. In addition, ADT was performed as neoadjuvant therapy and it did not affect rectal bleeding.

As VRS proposed by Wachter et al. varies in grade by TE, congested mucosa, UL, stricture, and necrosis, it is useful for the comprehensive evaluation of endoscopic findings of CRP [13]. VRS has been correlated with rectal GI toxicity caused by pelvic radiotherapy [20,21]. However, VRS is more sensitive compared to rectal bleeding score systems evaluated by clinical symptoms [13, 21]. A similar result was obtained in our study; no bleeding symptom was observed in $56.3 \%$ and $23.1 \%$ of patients with VRS 2 and VRS $\geq 3$, respectively.

In the present study, severity of endoscopic findings increased during 1 to 2 year(s) after treatment and improved over the 5 years of observation. There are only a limited number of reports about the long-term endoscopic evaluation of CRP. Goldner et al. performed proctosigmoidoscopy at 12 and 24 months in patients with prostate cancer who underwent EBRT (range 70 to $75 \mathrm{~Gy}$ ) and reported that VRS $\geq$ 1 was found in $64 \%$ and $62 \%$ of the patients at 12 and 24 months, respectively [21]. On the other hand, O'Brien et al. performed proctosigmoidoscopy in prostate cancer patients who underwent EBRT with $65 \mathrm{~Gy}$ and showed that the incidence of multiple TE in the rectum at 24 months was higher than that at 12 months and that it was lower at 36 months [22]. Furthermore, Goldner et al. performed a longterm follow-up with endoscopy at 12, 24, and 65 months and showed that the percentage of patients with VRS 1 increased and the percentage of patients with VRS 2 decreased with time [8]. The improving tendency of VRS over the course of a long-term follow-up was similar to the results of our study. In this study, no abnormal endoscopic finding was observed in $50 \%$ of the patients who presented with rectal bleeding of grade $\geq 1$ until the first year after BT. Increased severity of endoscopic findings was observed more often between 1 year and 2 years after BT. Delayed development of rectal mucosal change was observed compared to previous report [21]. This difference can be explained by the fact that EBRT + BT and BT were compared in our study, whereas previous studies evaluated the effects of EBRT alone; moreover, in our study, EBRT boost was initiated at the 4-8th week after BT.

Ippolito et al. performed endoscopy in prostate cancer patients treated with intensity-modulated radiation therapy or three-dimensional conformal radiation therapy, 1 year after treatment, and reported that the occurrence of grade $\geq 2$ rectal bleeding was $32 \%$ and $47 \%$ in patients with VRS $\geq$ 2 and VRS $\geq 3$, respectively, indicating the effectiveness of VRS in predicting rectal bleeding [23]. In the present study, as shown in the Kaplan-Meier curve, most rectal bleeding symptoms occurred between the 50th and 150th week. Hence, we examined whether it would be possible to determine the prognosis of CRP based on the endoscopic findings observed during this period. Our results showed the natural decrease of VRS over time in $31 \%(5 / 16)$ of patients with VRS $\geq 2$, as well as possible UL 1 improvement, while mild endoscopic findings (VRS 0-1) remained unchanged. No study report has examined factors affecting the recovery of endoscopic findings. Nevertheless, because the number of patients was limited in this study, more patients will be necessary for further investigation in the future. Although the effectiveness and safety of APC therapy have been demonstrated for rectal bleeding due to CRP $[4,24]$, patients using nonsteroidal anti-inflammatory drugs have shown the development of stenosis after treatment [25] as well as the occurrence of ulcer [26]. Therefore, the endoscopic findings at the occurrence of symptoms are deemed useful for considering treatment indications such as APC or HBO therapy.

\section{Conclusion}

While the incidence of rectal bleeding is low after BT for prostate cancer, EBRT boost increases its risk. Endoscopy was correlated with bleeding symptoms such as GI toxicity, and it allows an accurate evaluation of rectal mucosal damage. Endoscopy in the first 150 weeks after BT is useful for estimating the long-term prognosis of CRP.

\section{Competing Interests}

The authors declare that there are no competing interests regarding the publication of this paper.

\section{Acknowledgments}

We would like to thank Editage (http://www.editage.jp/) for English language editing.

\section{References}

[1] R. Siegel, D. Naishadham, and A. Jemal, "Cancer statistics for Hispanics/Latinos, 2012," CA: A Cancer Journal for Clinicians, vol. 62, no. 5, pp. 283-298, 2012.

[2] T. Matsuda, T. Marugame, K.-I. Kamo, K. Katanoda, W. Ajiki, and T. Sobue, "Cancer incidence and incidence rates in Japan in 2006: based on data from 15 population-based cancer registries in the Monitoring of Cancer Incidence in Japan (MCIJ) project," Japanese Journal of Clinical Oncology, vol. 42, no. 2, Article ID hyr184, pp. 139-147, 2012.

[3] V. Appalaneni, R. D. Fanelli, R. N. Sharaf et al., "The role of endoscopy in patients with anorectal disorders," Gastrointestinal Endoscopy, vol. 72, no. 6, pp. 1117-1123, 2010. 
[4] W. Tam, J. Moore, and M. Schoeman, "Treatment of radiation proctitis with argon plasma coagulation," Endoscopy, vol. 32, no. 9, pp. 667-672, 2000.

[5] T. J. Saclarides, D. G. King, J. L. Franklin, and A. Doolas, "Formalin instillation for refractory radiation-induced hemorrhagic proctitis.Report of 16 patients," Diseases of the Colon and Rectum, vol. 39, no. 2, pp. 196-199, 1996.

[6] M. A. Dall'Era, N. B. Hampson, R. A. Hsi, B. Madsen, and J. M. Corman, "Hyperbaric oxygen therapy for radiation induced proctopathy in men treated for prostate cancer," The Journal of Urology, vol. 176, no. 1, pp. 87-90, 2006.

[7] R. Kochhar, P. V. J. Sriram, S. C. Sharma, R. C. Goel, and F. Patel, "Natural history of late radiation proctosigmoiditis treated with topical sucralfate suspension," Digestive Diseases and Sciences, vol. 44, no. 5, pp. 973-978, 1999.

[8] G. Goldner, R. Pötter, A. Kranz, A. Bluhm, and W. Dörr, "Healing of late endoscopic changes in the rectum between 12 and 65 months after external beam radiotherapy," Strahlentherapie und Onkologie, vol. 187, no. 3, pp. 202-205, 2011.

[9] N. Oyama, Y. Aoki, H. Ito et al., "Alpha 1-adrenoceptor blocker may improve not only voiding but also storage lower urinary tract symptoms caused by ${ }^{125} \mathrm{I}$ brachytherapy for prostate cancer," ISRN Urology, vol. 2014, Article ID 140654, 8 pages, 2014.

[10] National Cancer Institute, National Cancer Institute Common Terminology Criteria for Adverse Events (CTCAE) v.4.0., http://evs.nci.nih.gov/ftp1/CTCAE/About.html.

[11] J. G. Price, N. N. Stone, and R. G. Stock, "Predictive factors and management of rectal bleeding side effects following prostate cancer brachytherapy," International Journal of Radiation Oncology, Biology, Physics, vol. 86, no. 5, pp. 842-847, 2013.

[12] L. H. Sobin and Ch. Wittekind, International Union Against Cancer. Prostate in Classification of Malignant Tumor, WileyLiss, New York, NY, USA, 2002.

[13] S. Wachter, N. Gerstner, G. Goldner, R. Pötzi, A. Wambersie, and R. Pötter, "Endoscopic scoring of late rectal mucosal damage after conformal radiotherapy for prostatic carcinoma," Radiotherapy and Oncology, vol. 54, no. 1, pp. 11-19, 2000.

[14] M. Buckstein, S. Kerns, K. Forysthe, N. N. Stone, and R. G. Stock, "Temporal patterns of selected late toxicities in patients treated with brachytherapy or brachytherapy plus external beam radiation for prostate adenocarcinoma," BJU International, vol. 111, no. 3, pp. E43-E47, 2013.

[15] M. Aoki, K. Miki, H. Sasaki et al., "Evaluation of rectal bleeding factors associated with prostate brachytherapy," Japanese Journal of Radiology, vol. 27, no. 10, pp. 444-449, 2009.

[16] K. Harada, H. Ishikawa, Y. Saito et al., "Risk factors for rectal bleeding associated with I-125 brachytherapy for prostate cancer," Journal of Radiation Research, vol. 53, no. 6, pp. 923929, 2012.

[17] S. K. Kang, R. H. Chou, R. K. Dodge et al., "Gastrointestinal toxicity of transperineal interstitial prostate brachytherapy," International Journal of Radiation Oncology, Biology, Physics, vol. 53, no. 1, pp. 99-103, 2002.

[18] D. Y. Gelblum and L. Potters, "Rectal complications associated with transperineal interstitial brachytherapy for prostate cancer," International Journal of Radiation Oncology, Biology, Physics, vol. 48, no. 1, pp. 119-124, 2000.

[19] K. Takeda, Y. Ogawa, H. Ariga et al., "Clinical correlations between treatment with anticoagulants/antiaggregants and late rectal toxicity after radiotherapy for prostate cancer," Anticancer Research, vol. 29, no. 5, pp. 1831-1834, 2009.
[20] T. G. Kim, S. J. Huh, and W. Park, "Endoscopic findings of rectal mucosal damage after pelvic radiotherapy for cervical carcinoma: correlation of rectal mucosal damage with radiation dose and clinical symptoms," Radiation Oncology Journal, vol. 31, no. 2, pp. 81-87, 2013.

[21] G. Goldner, B. Tomicek, G. Becker et al., "Proctitis after external-beam radiotherapy for prostate cancer classified by Vienna Rectoscopy Score and correlated with EORTC/RTOG score for late rectal toxicity: results of a prospective multicenter study of 166 patients," International Journal of Radiation Oncology, Biology, Physics, vol. 67, no. 1, pp. 78-83, 2007.

[22] P. C. O’Brien, C. S. Hamilton, J. W. Denham, R. Gourlay, and C. I. V. Franklin, "Spontaneous improvement in late rectal mucosal changes after radiotherapy for prostate cancer," International Journal of Radiation Oncology Biology Physics, vol. 58, no. 1, pp. 75-80, 2004.

[23] E. Ippolito, M. Massaccesi, C. Digesù et al., "Early proctoscopy is a surrogate endpoint of late rectal toxicity in prostate cancer treated with radiotherapy," International Journal of Radiation Oncology, Biology, Physics, vol. 83, no. 2, pp. e191-e195, 2012.

[24] J. J. Tjandra and S. Sengupta, "Argon plasma coagulation is an effective treatment for refractory hemorrhagic radiation proctitis," Diseases of the Colon and Rectum, vol. 44, no. 12, pp. 1759-1765, 2001.

[25] S. Taïeb, A. Rolachon, J.-C. Cenni et al., "Effective use of argon plasma coagulation in the treatment of severe radiation proctitis," Diseases of the Colon and Rectum, vol. 44, no. 12, pp. 1766-1771, 2001.

[26] T. Koessler, V. Servois, P. Mariani, E. Aubert, and W. Cacheux, "Rectal ulcer: due to ketoprofen, argon plasma coagulation and prostatic brachytherapy," World Journal of Gastroenterology, vol. 20, no. 45, pp. 17244-17246, 2014. 


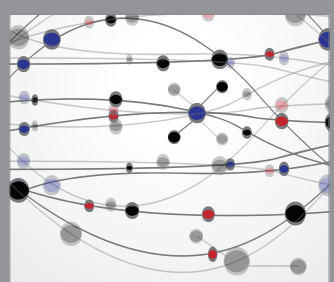

The Scientific World Journal
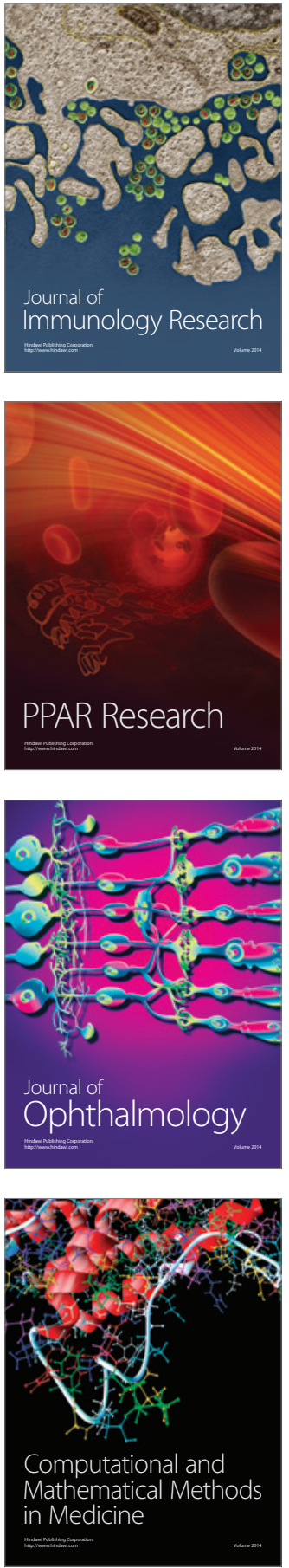

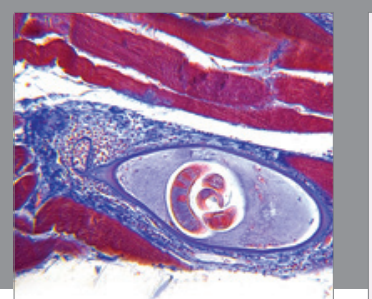

Gastroenterology Research and Practice

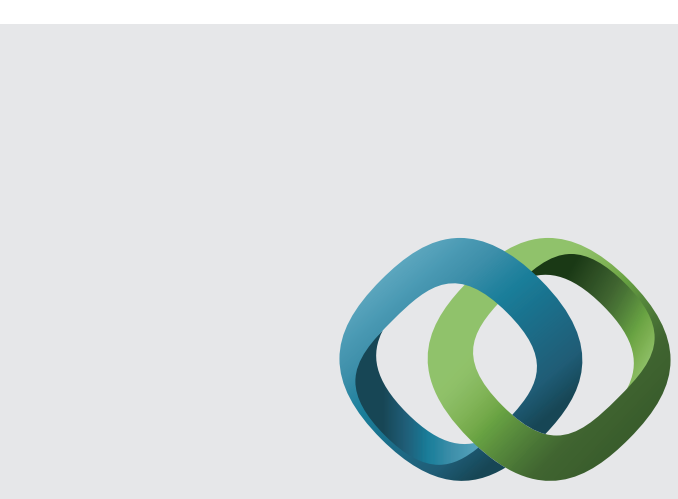

\section{Hindawi}

Submit your manuscripts at

http://www.hindawi.com
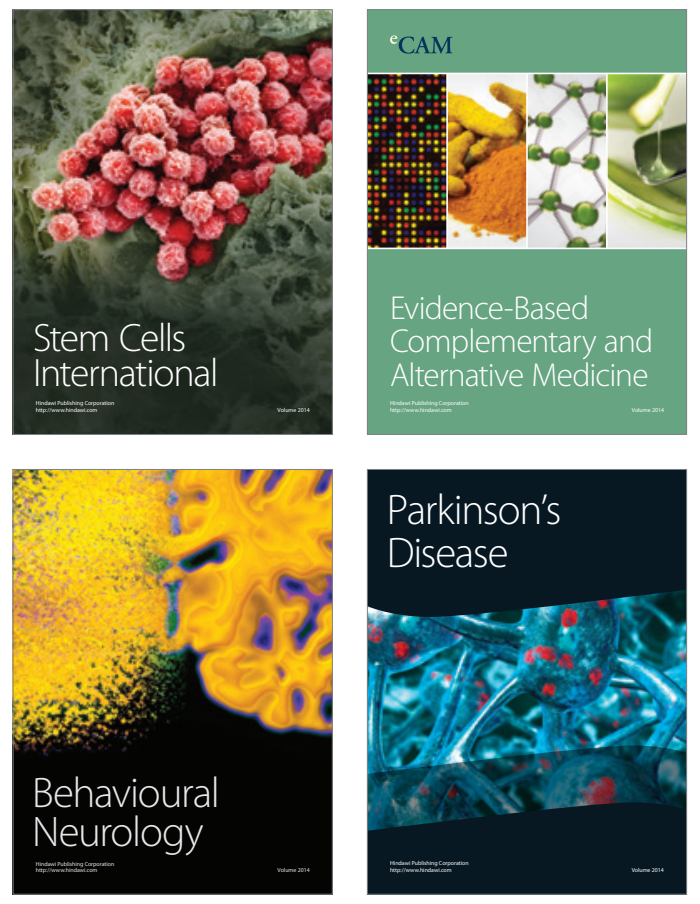
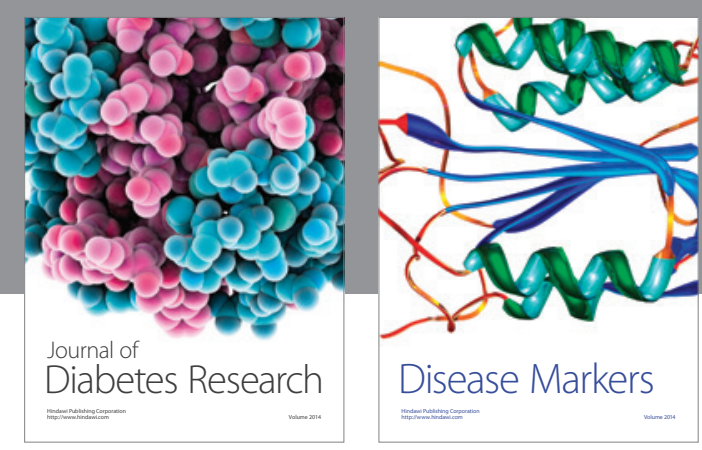

Disease Markers
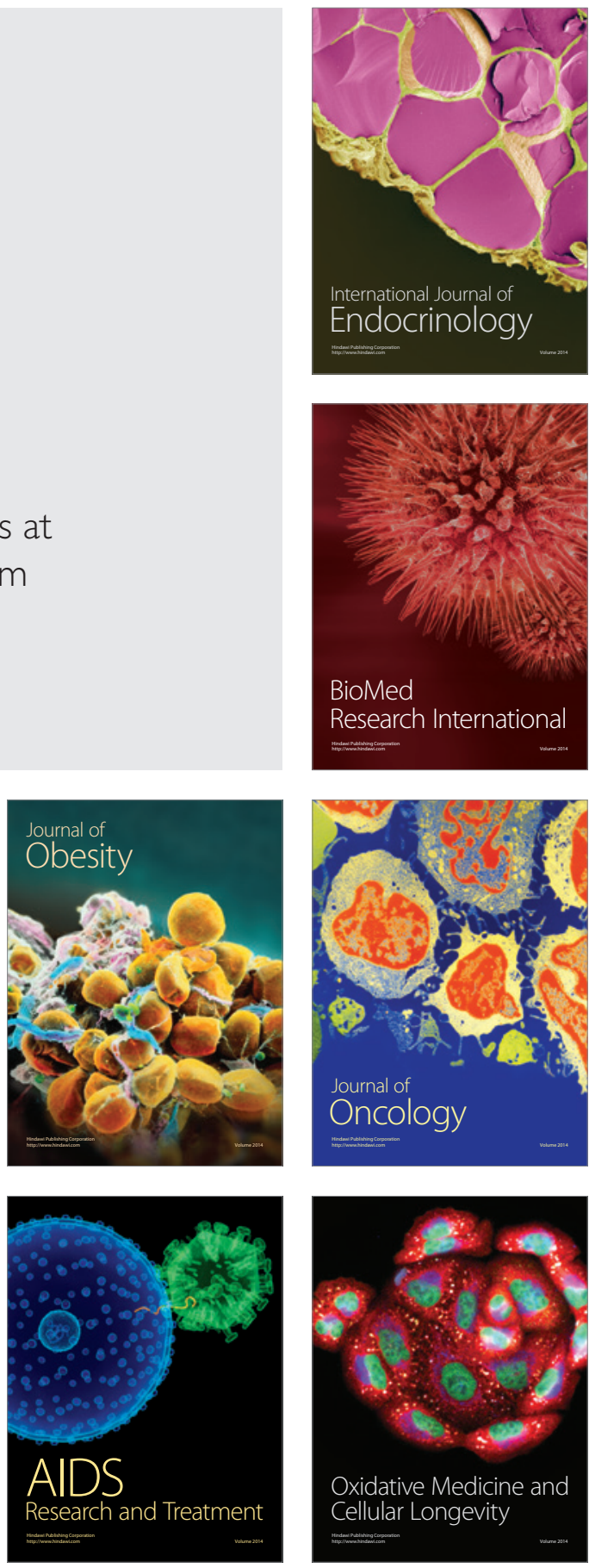